\title{
INSTRUMENTATION OF FOUR-POINT BENDING TEST DURING 4D COMPUTED TOMOGRAPHY
}

\author{
Daniel Kytýř $\check{R}^{a, *}$, Tomáš Fíla ${ }^{a}$, Petr Koudelka ${ }^{a}$, Ivana Kumpováa ${ }^{a}$, \\ Michal Vopálenskí ${ }^{a}$, LeOna VAVro ${ }^{b}$, Martin VAVro $^{b}$ \\ ${ }^{a}$ Czech Academy of Sciences, Institute of Theoretical and Applied Mechanics Prosecká 809/76, 19000 Prague 9, \\ Czech Republic \\ ${ }^{b}$ Czech Academy of Sciences, Institute of Geonics Studentská 1768, 70800 Ostrava-Poruba, Czech Republic \\ * corresponding author: kytyr@itam.cas.cz
}

\begin{abstract}
High-resolution time-lapse micro-focus X-ray computed tomography is an effective method for investigation of deformation processes on volumetric basis including fracture propagation characteristics of non-homogeneous materials subjected to mechanical loading. This experimental method requires implementation of specifically designed loading devices to X-ray imaging setups. In case of bending tests, our background research showed that no commercial solution allowing for reliable investigation of so called fracture process zone in quasi-brittle materials is currently available. Thus, this paper is focused on description of recently developed in-situ four-point bending loading device and its instrumentation for testing of quasi-brittle materials. Proof of concept together with the pilot experiments were successfully performed in a CT scanner TORATOM. Based on results of the pilot experiments, we demonstrate that crack development and propagation in a quasi-brittle material can be successfully observed in 3D using high resolution 4D micro-CT under loading.
\end{abstract}

KEYWORDS: in-situ loading device, four-point bending, 4D micro-CT, crack propagation.

\section{INTRODUCTION}

Proper characterization of damage processes in nonhomogeneous materials requires development of advanced experimental procedures and instrumentation of testing devices. One of currently investigated problems is evaluation of the so called fracture process zone (FPZ) [1] together with crack propagation in quasi-brittle building materials 2, e.g. sandstones or man-made cementitious composites. For this purpose, notched samples are typically submitted to flexural loading in standard loading frames. In case of optical or thermographical measurement, basic information about crack propagation can be obtained, but information about deformation processes and FPZ evolving within volume of the tested sample stays uncovered. For that reason, radiographical observation of the loading procedure capable of revealing microstructural variations in $3 \mathrm{D}$ is one of the most efficient methods [3].

To obtain relevant information about deformation and fracture behaviour of investigated quasi-brittle material, development of complex experimental procedure including design of a specialised loading device was necessary. We proposed a novel approach to four-point bending experiments during high-resolution micro-CT scans, which is based on vertical orientation of the investigated specimen, whose axis of rotations is identical with rotational axis of the CT scanner. This arrangement significantly reduces fluctuation of $\mathrm{X}$-ray attenuation during the imaging and thanks to smaller source-object distance higher resolution of the reconstructed $3 \mathrm{D}$ images can be achieved during the experimental procedure. Aside from technical details of the experimental device, proof of concept together with the preliminary results are presented in this paper.

\section{FRACTURE OF QUASI-BRITTLE MATERIAL}

Fracture of quasi-brittle materials is accompanied by formation and evolution of an inelastic zone around the propagating crack tip. This area referred to as the FPZ, which starts to appear at $\approx 75 \%$ of ultimate stress is responsible for the non-linear propagation of the crack in quasi-brittle materials 44. Typical deformation response to the loading of the quasi-brittle material together with scheme of FPZ is depicted in fig. 1] In this zone, the damage on several scale levels of material microstructure can be detected.

Since the FPZ is a non-linear zone of damage in front of the macroscopic crack propagating through the material, its influence on overall damage resistance of quasi-brittle structural elements has to be treated with respect to ratio of FPZ size to size of the assessed structural element. It has already been shown that size of the FPZ may be comparable to the material's basic constituents (e.g. the aggregates and voids) or it can be greater in extent by up to two orders of magnitude [5]. Here, X-ray micro-CT measurement plays crucial role in detection of the crack tip and FPZ propagation in the materials' microstructure. 


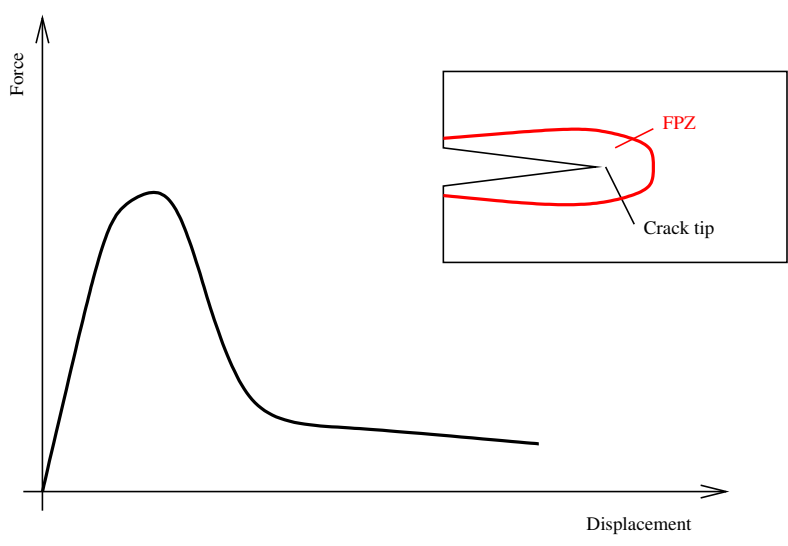

Figure 1. Typical $F-d$ curve of a quasi-brittle material subjected to flexural loading (left), schematic propagation of FPZ (right)

\section{INSTRUMENTATION}

Since 2013, the modular system for X-ray based observation of time-dependent processes in wide range of structural and functional materials has been continually developed to fulfill recent challenges of experimental mechanics and to keep all technologies of the system up-to-date. The system consists of the patented TORATOM imaging device (European patent no. EP2835631) and various loading device for specific experimental purposes.

\subsection{X-RAY IMAGING DEVICE}

In the current configuration, the device consists of two modular perpendicular imaging chains, which enables dual-source/dual-energy radiographical and tomographical experiments. Wide range of its applications is given by various $\mathrm{X}$-ray sources and detectors currently available. The first XWT 160 TCHR (X-RAY WorX, Germany) $160 \mathrm{keV}$ microfocus transmission tube with $1 \mu \mathrm{m}$ focal spot at target power of $3 \mathrm{~W}$ can be used for detailed analysis of materials with complex microstructure. The second high power XWT 240 SE (X-RAY WorX, Germany) 280 W reflection tube allows to analyse physically large samples or samples of materials with high attenuation of X-rays. Furthermore, it is possible to use different types of radiation imaging detectors - scintillating large-area flat panel detector, or various semiconductor pixelated detectors of the Medipix family [6, 7] with theoretically unlimited dynamic range, including the large area detector composed of $10 \times 10$ Timepix 2 assembly [8], fast devices with maximum possible frame rate of $200 \mathrm{fps}$ without compression in two-row/twocolumn configuration of Timepix chips [9], and also spectroscopic detectors. For the analysis presented in this paper, a combination of the XWT 160 source and XRD1622 (PerkinElmer, USA) scintillating large-area flat panel detector was used.

\subsection{IMAGE CORRECTION PROCEDURE}

It is a well known fact that the raw radiograms cannot be usually used for a high-resolution $\mathrm{CT}$ reconstruction procedure with sufficient quality of the reconstructed 3D image as they may suffer from stationary noise caused by the inhomogeneous characteristics of individual pixels, inhomogeneity of the background brightness caused by the intensity profile of the X-ray beam, and abnormal response of individual pixels (so called bad pixels). These effects then form different types of artifacts in the $3 \mathrm{D}$ reconstruction. For their elimination, the flat field correction [10] with equalization of the images and bad pixel correction were applied. These corrections were performed using the Torast in-house developed Python software (for a usage example see [11]). All the functions are available in a batch mode, allowing a comfortable processing of whole sets of projection images. New features are currently being integrated, such as the compensation of focal spot movement, elimination of residual gradients in the flat-field corrected images). In the future, the software will be integrated with the control software of the TORATOM device to enable advanced acquisition methods.

\subsection{LOADING DEVICE}

The concept for $4 \mathrm{D}$ micro-CT of the bending experiments was developed based on experience from measurements presented in [12, 13], where conventional three-point bending tests (see fig. 2 left) were employed. Although it was possible to obtain quantification of the crack-tip propagation processes, the horizontal orientation of the sample perpendicular to the rotational axis of the CT scanner together with the loading supports covering parts of the radiograms caused limitations in terms of contrast and resolutions.

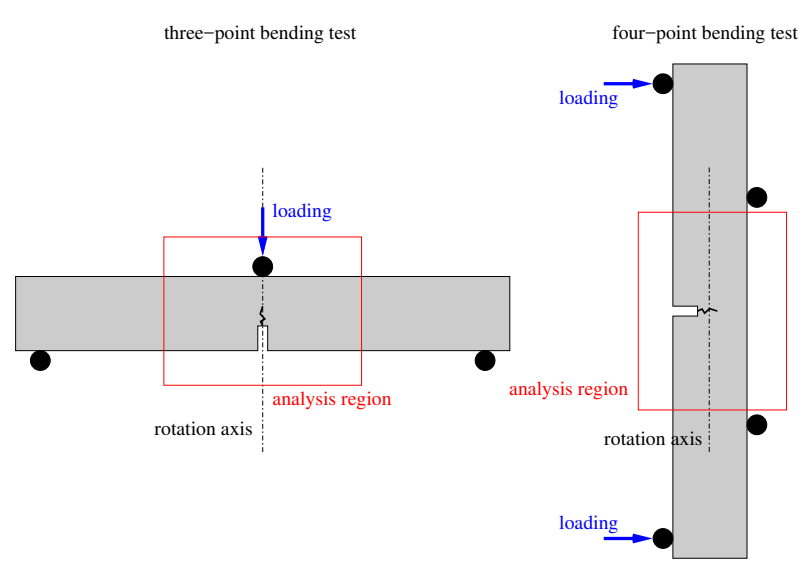

Figure 2. Basic principles of the loading scenarios

These disadvantages were overcome by employment of four-point bending setup, where the sample is oriented vertically with its longitudinal axis identical with the rotational axis of the CT scanner (see fig. 2 right).

The newly developed device (Czech national patent No. PV2018-28 pending) is composed of three main 


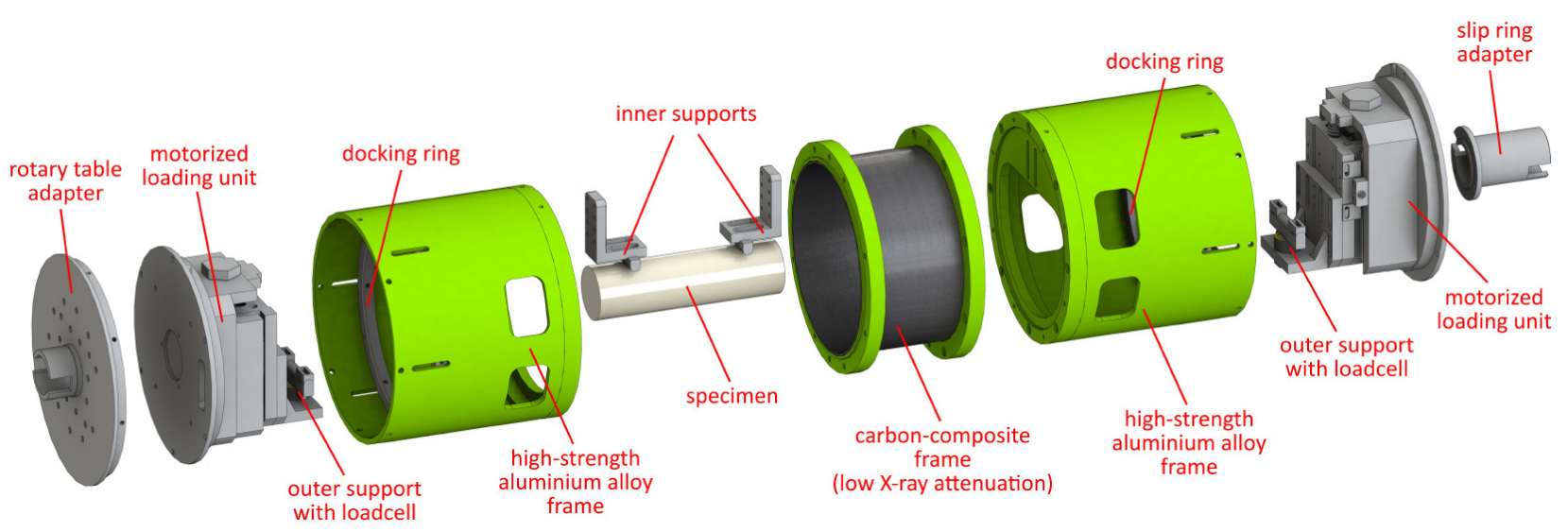

Figure 3. Components of the 4PB loading device

components: a pair of motorized loading units with integrated movable outer supports, a pair of a stationary inner supports, and the cylindrical load bearing frame housing the loaded specimen together with the components of the loading device. The design of the device in presented in fig. 3

The loading device is during the radiographically observed loading sequence oriented vertically and fixed on rotary stage of the CT scanner. To ensure high contrast in the acquired radiograms, the part of the loading frame located inbetween the inner supports was manufactured from carbon fiber composite (T700S carbon fibers, MTM57 series epoxy resin) with low attenuation of X-rays with the nominal thickness $1.95 \mathrm{~mm}$. The outer movable supports are integrated with driving units with precision captive stepper linear actuators (23-2210, Koco Motion DINGS, USA) and precision linear guideways (MGW12, Hiwin, Japan). Both driving units are independent and equipped with linear encoders with resolution $1 \mu \mathrm{m}$ (LM10, Renishaw, UK) for highly precise positioning. Each driving unit is also equipped with individual load cell (LCM300, Futek, USA) for force measurements on the outer supports. The maximum load capacity of the device is $1500 \mathrm{~N}$ per single support. Position accuracy and repeatability in single-direction loading is better than $10 \mu \mathrm{m}$ with sensitivity better than $1 \mu \mathrm{m}$.

\subsection{EXPERIMENTAL PROCEDURE}

The batches of specimens in shape of a cylinder with diameter $D=36 \mathrm{~mm}$ and length $L=195 \mathrm{~mm}$ were drilled out from the Godula sandstone, Kocbeře sandstone, and Mšené sandstone that all represent traditional building and decorative natural stones widely used in masonry for about a thousand years 14 . The core drilling was carried out parallel to the sandstone bedding planes. In central part of the sample, a chevron notch was prepared using a circular diamond blade. The specimen was subjected to four-point bending loading sequence performed as a force-driven experiment to ensure uniform load distribution on the supports (outer supports span $L_{\text {out }}=180 \mathrm{~mm}$, inner supports $\left.\operatorname{span} L_{i n}=150 \mathrm{~mm}\right)$. Experiments were con- trolled by the modified LinuxCNC software running on a real-time kernel [15]. During the experiment, displacement was interrupted at six discrete loading steps, where radiographical imaging was performed. In the loading sequence, one load-step was performed during the material's hardening phase, one load-step at the ultimate-stress point (peak force reached was approx. $325 \mathrm{~N}$ ), and four load-steps were performed during the post-peak softening phase. The reconstructed image data from the notch region with resolution better than $20 \mu \mathrm{m}$ showing evolution of the crack and the related changes in microstructure are depicted in the fig. 4

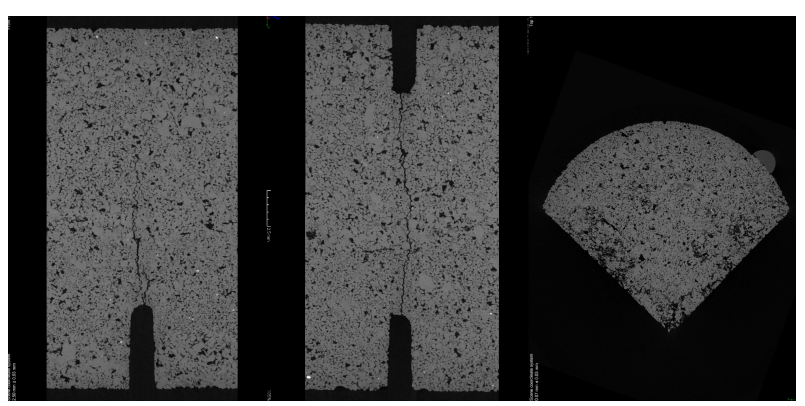

FiguRE 4. Visualization of damaged sample in three perpendicular planes based on the reconstructed microCT data corresponding to displacement $320 \mu \mathrm{m}$

\section{Conclusions}

Proof of concept based on the pilot experiments was successfully performed in a micro-CT scanner. Based on these experiments, it was shown that crack development and propagation in a quasi-brittle material can be observed in 3D using in-situ loading procedure and high resolution 4D micro-CT imaging. Arrangement of the new experimental setup together with higher stiffness of the loading device allows to interrupt the loading procedure at any point of the material's $F-d$ curve without the risk of sudden collapse of the sample. Concept of vertically oriented sample also allows to increase signal to noise ratio in the acquired projections to achieve higher contrast in the reconstructed $3 \mathrm{D}$ images. 


\section{ACKNOWLEDGEMENTS}

The research has been supported by the European Regional Development Fund in frame of the project Com3dXCT (ATCZ38) in the Interreg V-A Austria - Czech Republic programme and by Operational Programme Research, Development and Education in project INAFYM - Engineering applications of microworld physics (CZ.02.1.01/0.0/0.0/16_019/0000766).

\section{REFERENCES}

[1] J. Klon, V. Vesely. Modelling of size and shape of damage zone in quasi-brittle notched specimens analytical approach based on fracture-mechanical evaluation of loading curves. Frattura ed Integrita Strutturale 11(39):17-28, 2017. DOI:10.3221/IGF-ESIS.39.03

[2] V. Vesely, Z. Kersner, I. Merta. Quasi-brittle behaviour of composites as a key to generalized understanding of material structure. Procedia Engineering 190:126-133, 2017. DOI:10.1016/j.proeng.2017.05.317.

[3] L. Skarzynski, J. Suchorzewski. Mechanical and fracture properties of concrete reinforced with recycled and industrial steel fibers using Digital Image Correlation technique and X-ray micro computed tomography. Construction and Building Materials 183:283-299, 2018. DOI:10.1016/j.conbuildmat.2018.06.182.

[4] T. Anderson. Fracture Mechanics: Fundamentals and Applications. Third Edition. CRR Press, 2004.

[5] V. Vesely, Z. Kersner, J. Nemecek, et al. Estimation of fracture process zone extent in cementitious composites. Chemicke Listy 104(15 SPEC):s382-s385, 2010.

[6] J. Jakubek. Data processing and image reconstruction methods for pixel detectors. Nuclear Instruments and Methods in Physics Research Section A: Accelerators, Spectrometers, Detectors and Associated Equipment 576(1):223-234, 2007. DOI:http://dx.doi.org/10.1016/j.nima.2007.01.157

[7] E. Gimenez, V. Astromskas, I. Horswell, et al. Development of a Schottky CdTe Medipix3RX hybrid photon counting detector with spatial and energy resolving capabilities. Nuclear Instruments and Methods in Physics Research, Section A: Accelerators, Spectrometers, Detectors and Associated Equipment 824:101-103, 2016. DOI:10.1016/j.nima.2015.10.092.
[8] I. Kumpova, D. Vavrik, T. Fila, et al. High resolution micro-CT of low attenuating organic materials using large area photon-counting detector. Journal of Instrumentation 11(2), 2016. DOI:10.1088/1748-0221/11/02/C02003.

[9] D. Kytyr, P. Zlamal, P. Koudelka, et al. Deformation analysis of gellan-gum based bone scaffold using on-the-fly tomography. Materials and Design 134:400-417, 2017. DOI:10.1016/j.matdes.2017.08.036

[10] J. Rowlands, Y. J. Flat panel detectors for digital radiography. In Handbook of Medical Imaging, Vol. 1: Physics and Psychophysics. SPIE Press, 2000.

[11] M. Vopalensky, D. Vavrik, I. Kumpova. Optimization of the X-ray tube voltage with respect to the dynamical resolution in radiography and tomography. NDTnet Journal 2018-02, 2018.

[12] L. Vavro, K. Soucek, D. Kytyr, et al. Visualization of the evolution of the fracture process zone in sandstone by transmission computed radiography. Procedia Engineering 191:689-696, 2017. DOI:10.1016/j.proeng.2017.05.233.

[13] I. Kumpova, M. Vopalensky, T. Fila, et al. On-the-fly fast X-ray tomography inspection of the quasi-brittle material three point bending test. 2016 IEEE Nuclear Science Symposium, Medical Imaging Conference and Room-Temperature Semiconductor Detector Workshop 2017-January, 2017. DOI:10.1109/NSSMIC.2016.8069950

[14] M. Vavro, L. Vavro, P. Martinec, K. Soucek. Properties, durability and use of glauconitic Godula sandstones: a relatively less known building stone of the Czech Republic and Poland. Environmental Earth Sciences 75(22), 2016. DOI:10.1007/s12665-016-6248-3

[15] V. Rada, T. Fila, P. Zlamal, et al. Multi-channel control system for in-situ laboratory loading devices. Acta Polytechnica Proceedings CTU Proceedings 18:15-19 2018. DOI:10.14311/APP.2018.18.0015 